\title{
Heuristic-based neural networks for stochastic dynamic lot sizing problem
}

\author{
Ercan Şenyiğit ${ }^{\mathrm{a}}$, Muharrem Düğenci ${ }^{\mathrm{b}}$, Mehmet E. Aydin ${ }^{\mathrm{c}}$, Mithat Zeydan ${ }^{\mathrm{a}}$, \\ ${ }^{a}$ Erciyes University, Dept. of Industrial Engineering, Kayseri, Turkey \\ ${ }^{b}$ Karabük University, Dept. of Industrial Engineering, Karabük, Turkey \\ ${ }^{c}$ University of Bedfordshire, Dept. of Computer Science and Technology, UK
}

\begin{abstract}
Multi-period single-item lot sizing problem under stochastic environment has been tackled by few researchers and remains in need of further studies. It is mathematically intractable due to its complex structure. In this paper, an optimum lot-sizing policy based on minimum total relevant cost under price and demand uncertainties was studied by using various artificial neural networks trained with heuristic-based learning approaches; genetic algorithm (GA) and bee algorithm (BA). These combined approaches have been examined with three domainspecific costing heuristics comprising revised silver meal (RSM), revised least unit cost (RLUC), cost benefit (CB). It is concluded that the feed-forward neural network (FF-NN) model trained with BA outperforms the other models with better prediction results. In addition, RLUC is found the best operating domain-specific heuristic to calculate the total cost incurring of the lot-sizing problem. Hence, the best paired heuristics to help decision makers are suggested as RLUC and FF-NN trained with BA.
\end{abstract}

Keywords: Stochastic lot-sizing, Feed-Forward Neural Networks, Bee Algorithm, Genetic Algorithms, Taguchi methods.

\section{Introduction}

Lot-sizing problems have been studied with various respects for a long time whilist keeping the emphasis on modelling and optimisation of deterministic versions, which are known with the NP-Hard nature $[1,2,3]$ and usually handled with heuristic methods in-line with WagnerWhitin (WW) approach $[4,5,6]$. On the other hand, the real world versions of these problems are not as static and deterministic as modelled and handled in these ways, but, are rather dynamic and subject to probabilistic processes. That makes the problem type hard to model in easily solvable mathematical structures due to the complexity and uncertainty issues.

The stochastic dynamic lot-sizing (SDLS) problem can be formulated, in analytical or simulation models, either by assuming a penalty cost for each stock out and unsatisfied demand or by minimising the ordering and inventory costs subject to satisfying some customer service-level criterion. The analytical modelling approach is most frequently encountered in particular stochastic programming, where these models tackle only one type of uncertainty and assume a simple production system structure. Exact analytical solutions can only be developed, when the model is adequately simple. Further numbers of uncertain inputs/parameters escalate the complexity of the problems to an un-handlable state with analytical models. Due to the limitations given rise by stochastic/uncertain nature of controlling parameters in dynamic lot sizing problem (DLSP), heuristic approaches are preferred, rather than analytical models, in the solving larger-scale DLSP instances. 
Companies mostly work on a rolling horizon basis to form production plans consistent with new information on demand and prices which are usually uncertain and may only be knownsometimes partially over the forecast window. Since lot-sizing problem under uncertain demand and price conditions is so complex and mathematically intractable, generally, simulation techniques are used for obtaining good and feasible solutions. Simulation results have been accepted in finding total relevant cost as a real result. To reach the real total relevant cost, stochastic lot-sizing decision process under uncertain demand and price simultaneously using simulation was modelled by Manikas et al.[7] and Şenyiğit and Erol [8]. In this research, both price and demands are considered uncertain and assumed to be stochastic. On the other hand, among the heuristic approaches, artificial neural networks become very useful popular in modelling such ill-structured and/or highly complex problems, therefore stands promising to tackle SDLS problem. In fact, artificial neural networks with/without other metaheuristic approaches such as genetic algorithm have been interested in by recent research on solving various combinatorial optimization problems $[3,5,9]$.

This paper reports the attempts of solving SDLS problems with using a variety of artificial neural networks (ANN) trained with metaheuristic algorithms, namely genetic algorithms and bees algorithm, using Taguchi experimental design patterns in experimentation. To the best of authors' knowledge, Taguchi design patterns have not been used to compare the performance of domain-specific heuristics. Furthermore, ANN trained with Bee algorithms have never been used in solving lot sizing problems. The data used for training and testing purposes is gathered from the simulation model of Şenyiğit and Erol [8] instead of an analytical solution. In the rest of the paper, the background of SDLS problem is introduced in the second section, while the novel approaches employed are revealed in section three and four. The experimental study is provided in section five and conclusions are indicated in section six.

\section{Stochastic dynamic lot-sizing}

Lot sizing studies have attracted so much interest in the literature since it is an indicative problem used to model various real world production/stock management problems. The main objective is to determine the periods of production, with minimum costs including setup and inventory costs, in which the product quantities to be produced in order to satisfy demand. Efficient lot sizing requires efficient decision making in order to minimize the overall cost since these decisions have crucial impact on production and inventory system [3, 10]. Wagner-Whitin algorithm (WW) is known as the key methodology in which deterministic lot sizing problems are optimally solved [11]. However, the approach remains highly complex and very difficult to implement $[12,13]$. Therefore, numerous alternative methods to solve lot sizing problems have been developed besides various improvements achieved for WW [1, 3, 6, 14]. Silver and Meal (SM) and Part Period Balancing (PPB) methods are two leading approaches among these alternative methods [15, 16]. The analytical methods proposed usually consider certainty in key inputs (demand, price etc.) of the problem. However, once uncertainty is introduced into the model, it escalates to high complexity, which makes the problem hard to model in conventional ways. Then, as the probabilities play vital roles in calculations, the models turn to be stochastic. Monte-Carlo simulation implementing heuristic approaches remains the easiest way to go for robust problem solving, where simulation modelling complicates the process and leads to practical overheads [8]. In this paper, stochastic dynamic lot sizing problems with uncertain demand and price conditions have been considered; the simulation model and environment introduced by Şenyiğit and Erol [8], are used. 


\section{Artificial neural networks for stochastic dynamic lot-sizing}

SDLS problems are mainly modelled using either analytical or heuristic methods such as artificial neural networks, which are based on and/or assited by simulation. The main aim is to determine the best model in which optimum/near-optimum lot-sizes are identified subject to the environmental circumstances. Artificial neural networks (ANN) and genetic algorithms have been used in studying this sort of problems with various aims and scopes [3,17]. The durability of the ANN against noises, the fundamental characteristics of Von Neumann design reflected on the networks, and the need for sufficient, rather than detailed, knowledge about the problem are the strengths of ANNs in modelling dynamic and complicated problems such as determining the lot sizing [18]. Few studies were come acorss within the literature implementing ANN for solving lot sizing problems [9, 19]. Gaafar and Choueiki[5] applied an ANN model for a single item lot sizing problem, while Megala and Jawahar [9] proposed genetic algorithm and Hopfield neural network to solve the dynamic lot sizing problem with a capacity constraint and discount price structure.

In this study, multi-layer feed-forward neural network (FF-NN) has been chosen for the purpose of modelling SDLS problems with various training strategies owing to its maturity and simplicity in modelling. The FF-NN model is supported with a set of learning algorithms for training purposes and a simulation for data cultivation. Figure 1 depicts the system architecture in which the FF-NN models are configured, trained and tested alongside a simulation module. Since the probabilistic and variable properties of the system can easily be developed and handled using simulation, the data sets for training and testing FF-NNs are generated using simulation. The FF-NN models are configured as 3-layer feed-forward neural networks, where a learning algorithm is selected from the Learning Algorithms Base and applied. Here, three algorithms are included in the learning algorithms base: back propagation (BP), genetic algorithm (GA) and bee algorithm (BA). BP is the classical gradient searchbased learning algorithm calculates the error of the system and propagates it back to the weights of the connections of the network. On the other hand, both GA and BA are population based heuristic search algorithms used for optimisation purposes. The aim of using them in this study is to optimise the weights of the connections form the network so that the FF-NN model can predict the cost of lot-sizing system with minimum error. Once a learning algorithm is preferred, the configuration of FF-NN model is completed, then, the model is trained using the samples included in training data-set fetched from the Data Set unit, where the simulation results sampled in. The training phase is conducted until a certain level of learning is achieved. After that, another data-set is retrieved to test FF-NN's performance.

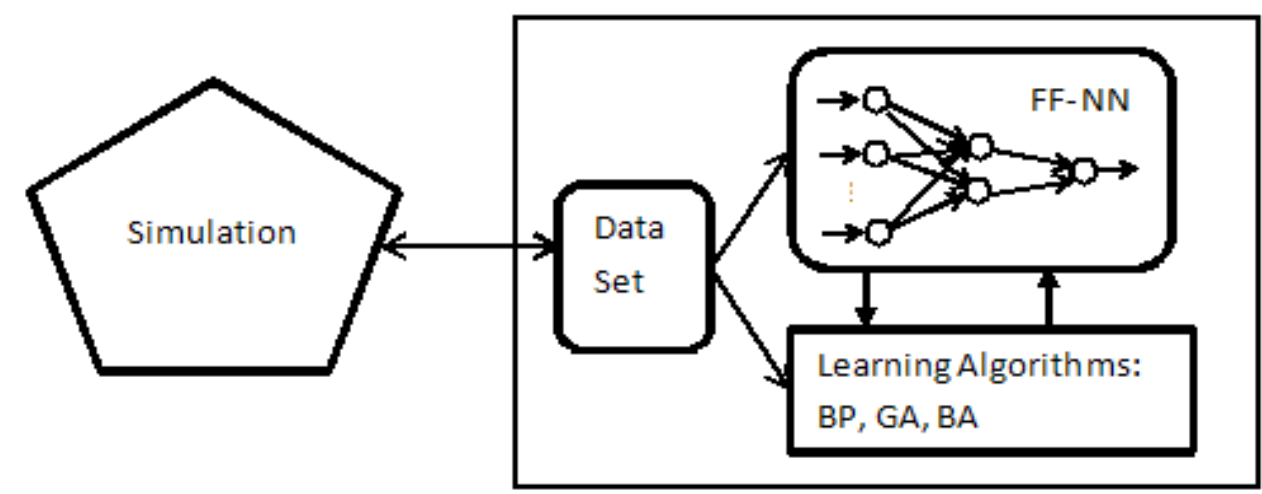

Figure 1: The complete system architecture 
As declared before, the main purpose of this study is to find the optimal lot-size (amount of order) that minimizes total relevant cost under price and demand uncertainties. The cost study was carried out comparatively to identify which heuristic approach for costing would be most appropriate and relevant in performance analysis. Using simulation results, costing heuristics/techniques known as revised silver meal (RSM), revised least unit cost (RLUC), cost benefit (CB) have been chosen [8]. Then, the following four modelling approaches, called Taguchi Design of Experiment (TDOE), FF-NN model trained with back propagation (BP), FF-NN model with GA and FF-NN with BA, have been implemented to solve SDLS problems. At the first stage, CB, RLUC, RSM costing methods are compared with one another with respect to the minimum total relevant cost based on simulation results using TDOE approach. As a result, RLUC method is selected for evaluation since it provides the higher correlation and lower error. Then, the problem is studied with TDOE starting with the modelling information to be considered as the system inputs with degrees of freedom, where each set of them reflects a unique state of the problem. Once identified, the set of inputs are fed in to the neural network models to find out system's response/output. Since the ultimate objective is to predict the lot-sizes with minimum error, the problem is modelled as a minimisation problem subsequently; the aim is set to "the smaller the difference is the better". Afterwards, Taguchi orthogonal arrays are considered to be used as sampling templates for setting up experimentation needed for both training and testing sets of FF-NN models. Once selected, then the BP, GA and BA models are trained and tested accordingly. Use of Taguchi OA's for sampling and collecting training sets of ANN's has been proposed by Öztemel and Aydin [20].

The distinctive part of this study is to use variable and stochastic price and demands. It is assumed that the demand in each period is normally distributed, where the mean rate of demand and price vary from period to period. The coefficient of variation is constant over time to use cumulative distribution of demand [21]. It is a well-known and widely-used assumption in the literature $[8,21,22,23,24]$. Also, price is a uniform random variable for ease of understanding as it is assumed in Manikas et al. [7]. Unit holding cost does not depend on purchasing price. Li et al [25] determined the quantities that have to be re-manufactured at each period to minimise the total relevant cost. Brandimarte [26] proposed a heuristic solution approach for dynamic lot-sizing problem production plans which were applied in a realistic rolling horizon framework.

The assumptions in this work are analogous to Bookbinder and Tan [21] with some differences. We assumed that purchasing price is non-stationary and stochastic as demand is. The total relevant cost is calculated as the sum of costs consisting of purchasing cost, set-up cost, holding cost, and lost sale cost. The following assumptions are used in modelling nonstationary stochastic inventory system in the simulation of this study, which are apparently more realistic.

i. The demand and price of raw material for periods are mutually independent random variables. Demand sizes are assumed to be normal random variables, and prices are uniform random variables.

ii. Expected value for demand size and price are required before making lot sizing decisions.

iii. Non-stationary demand and price patterns are permitted.

iv. Lot sizing decisions are made on a rolling horizon basis.

v. Partial or complete lost sale are permitted.

vi. Order lead time is zero. 


\section{Learning with Genetic algorithms (GA) and Bees Algorithm (BA)}

The need for an improved learning approach over the fundamental back-propagation algorithm is due to its coarse-grained approximation to find the most appropriate weights so as to estimate the outputs as accurate as possible. Various gradient-based approaches have been proposed to overcome this shortcoming. Among these are the metaheuristic approaches such as genetic algorithms [27,28,29], particle swarm optimisation and artificial bee colony algorithms [30,31,32]. In this paper, we report the performance of two attempted approaches: genetic algorithm, and bee algorithm.

Genetic algorithms (GA) are of very well-known metaheuristic approaches used in optimisation, which have a strong record of success in solving difficult problems such as NPHard ones. That encourages researchers to use it for solving various problems. Among these is the training of ANN models, which appears as the problem of finding the optimum set of weights, which makes the ANN models best-learned and interpolate/extrapolate values expected. Chan et al [27,28] have proposed a cutting edge GA approach for training ANN models, while an early and comprehensive review on evolutionary neural networks is provided by Yao [29].

Artificial bee colony-based algorithms are of swarm intelligence algorithms developed recently, which are inspired of the social behaviour of the natural bee colonies [33]. This family of algorithms has been successfully used for various applications such as modelling on communication networks, manufacturing cell formation, training artificial neural networks $[34,35,36]$. The main idea behind a simple bee colony optimisation algorithm is to follow the most successful member of the colony in conducting the search. The scenario followed is that once a bee found a fruitful region, then it performs the waggle dance to communicate to the rest of the colony so that a number of peer-bees come to that area for a better collective search of food. Inspired of this natural process, bee algorithms are implemented for efficient search methodologies borrowing this idea to direct the search to a more fruitful region of the search space. That would result a quicker search for an appropriate solution to be considered as a neat near-optimum. The detailed information on the bee algorithm (BA) attempted in this research can be found in Düğenci [32].

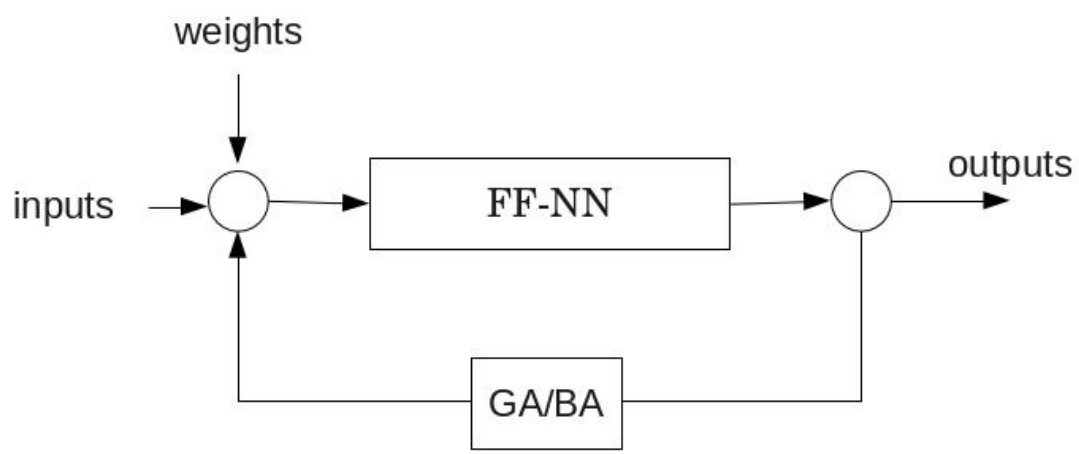

Figure 2: Learning procedures with heuristic algorithms 
Figure 2 sketches the idea in which FF-NN model is trained. The model is provided with two sets of entries; inputs and initial weights. The set of inputs is identified with the description of the problem while the set of initial weights determined in a semi-random scheme in which the model is trained with a back-propagation (BP) algorithm to identify the weights, and then, each weight is re-generated as follows:

Let $W$ be the set defined as $W=\left\{w_{i}|i=0, . .| W \mid,\right\}$, where $w_{i}$ is weight $i$ included in the weight set. Let also $\Delta$ be the set of differences defined as $\Delta=\left\{\delta_{i}|i=0, . .| W \mid,\right\}$ noting that the differences should not be huge, but challenging enough. Each particular weight, $w_{i} \in W$, represents the weight of a particular connection between any lower level neuron and an upper level neuron, is regenerated conforming the following rule:

$$
\left(w_{i}-\delta_{i}\right) \leq \bar{w}_{i} \leq\left(w_{i}+\delta_{i}\right)
$$

where $\bar{w}_{i}$ is the regenerated weight. Once regeneration is completed, the new set of weights is represented with $\bar{W}$. Then, the weights are assigned to corresponding connectors of neural network model. Further training with any chosen heuristic algorithm will operate on this set of weights to improve the performance of FF-NN. The next step to fulfil further training is to generate a population of solutions. Let $P$ be the population of $|P|$ individual solutions, where each individual solution is made of a complete set of weights, which is $\bar{W}$ at the initial stage. As the whole population will be a set of different solutions, each will be recognised with its index, where $\bar{W}_{p} \in P$ is the $p^{\text {th }}$ solution in the population. The training is conducted as a particular search for the best set of weights, $\hat{W}$, where the quality of solution plays the role of the main performance measure. To be in-line with the context of evolutionary computation, we call this performance measure as fitness function. The fitness function of this case is the total error of FF-NN model in estimating the total cost of the whole system subject to given set of weights and the input sets included in training data set. The fitness function is defined as $f:(I+\bar{W}) \rightarrow \mathfrak{R}$, where $I$ is the input set taken from training data set. The fitness of individual solution $p$ is represented as $f_{p}$ and calculated as follows:

$$
f_{p}=f\left(I_{p}, \bar{W}_{p}\right)=\sum_{j \in J} \varepsilon_{j}
$$

where $\varepsilon_{j}$ is the error of output neuron $j$ supposing there are $J$ numbers of output neurons in the FF-NN model.

Given the population $P$, a genetic algorithm will operate through $G$ generations in which a set of genetic operators, including crossover, mutation, and selection operators, are used to develop the new population. A two-point crossover, a simple mutation and a binary tournament selection are employed through a generational genetic algorithm. The parametric details are provided in Section 5.3.

The bee algorithm is also implemented based on abovementioned definitions. In this case, the population $P$ consists of bees, where each bee represents a particular solution made of a complete set of weights. The whole population, so-called hive in bee colony context, is broken down into groups of bees, where each group fulfils a particular set of duties in harmony for the purpose of keeping diversity while evolving the population towards the best solution. Let $N=|P|$ be the size of the whole bee colony, which is also called scout bees, 
and $M$ be the number of sites visited/searched by the scouts. There are $E$ numbers of sites found very valuable to search in depth, which are called elite sites and considered as a subset of the searched sites, $E \subseteq M$. The total number of bees recruited on elite sites is $n_{E}$, which is calculated as $n_{E}=\sum_{i \in E} e_{i}$, where $e_{i}$ is the number of bees recruited per elite site. The nonelite sites, $M-E$, are also assigned with bees from the colony, which can be represented as $n_{M-E}$ and calculated as $n_{M-E}=\sum_{i \in M-E} \bar{e}_{i}$, where $\bar{e}_{i}$ is the number of bees deployed on non-elite site $i$. In addition to abovementioned sites, a number of bees are assigned to carry on random search around the hive in order to keep diversity and keep up with aspiration. The number of this group of bees is represented with $n_{o}$ such that $N=n_{E}+n_{M-E}+n_{o}$. The new-born bees are generated using a neighbourhood function, $N F: W_{i} \rightarrow \bar{W}_{i}$, which randomly alternates a new set of weights. In this particular case, $N F$ is implemented to revise the weights with $w_{i}=\bar{w}_{i}+\rho \gamma$, where $\rho$ is a random number varies in the range of $[-1,1]$ while $\gamma$ is the granularity coefficient, which normalizes the amount of change. $N F$ is also used as the mutation operator in GA implementation. The BA first initialises all abovementioned parameters and determines the fitness of each bee within the colony, then sorts them in a particular order. Afterward, it repeats (i) identifying the elite sites and sending addition teams of bees to each one, (ii) identifying non-elite sites, and recruiting corresponding teams on each, (iii) generates a set of new-born bees, and finally (iv) evaluating the fitness of every bee. This set of actions is iterated up to satisfaction of a predefined criterion.

\section{Experimental Results}

In this paper, the multi-period single-item lot sizing problem under stochastic environment with demand and price uncertainties is solved with FF-NN models. The uncertainties considered with price and demand, are in terms of raw material, which makes the problem the same as stochastic lot sizing problem. First of all, three simulation models have been developed using ARENA simulation package implementing three problem solving heuristics; Revised Least Unit Cost (RLUC), Revised Silver-Meal (RSM) and Cost Benefit (CB), respectively. For the purpose of verification, the simulated models have been run with some realistic data and cleared of verification. Both RLUC and RSM heuristics are the modified versions of well-known heuristics called Silver-Meal and Least-Unit Cost while the CostBenefit is based on a cost-benefit evaluation at decision points [8]. The simulation models have been run and results collected for all three heuristics for comparative purpose. The comparisons are conducted using TDOE method, which resulted in that the outperforming heuristic is found as RLUC. Following these initial experimentations, we generated a data set of totally 129 values (102 used for training and 27 used for testing) for configuring, training and testing TDOE, BP, GA, and BA models. Following is the details provided.

\subsection{Taguchi Design of Experiment (TDOE)}

An experimental design method has been developed by Japanese engineer G. Taguchi, which empowers the fractional factorial design approach. It is based on analysis of variances (ANOVA), which tries to reveal the main causes of variations in the experimentations. The method starts with identification of the problem in which all aspects of the problem is analysed and all possible factors, which might have impact of the results are considered. Then a list of factors effective on the process, called control factors, is developed with degrees of freedom. For this particular problem, the control factors are tabulated in Table 1 with 
corresponding possible levels. The aim of SDLS problem is to minimise the total system cost calculated with RLUC, which can be considered as the main objective of model.

Table 1. Control factors and levels for the experimental design

\begin{tabular}{llccc}
\hline Factor & Description & Level1 & Level2 & Level3 \\
\hline A & Forecast Window (Periods) & 7 & 14 & 30 \\
B & Coefficient of variation of demand size & 0.1 & 0.2 & 0.3 \\
C & Coefficient of variation of price & 0.1 & 0.2 & 0.3 \\
D & Minimum order quantity (Tonnes) & 100 & 200 & 500 \\
E & Set-up cost (\$) & 200 & 300 & 500 \\
F & Holding cost $(\$)$ & 0.5 & 1 & 2 \\
G & Unit lost sale cost $(\$)$ & 3 & 5 & 8 \\
\hline
\end{tabular}

The factors in Table 1 are labelled with A to G and described, accordingly, and indicated all possible values to be taken at each level. The primary analysis indicates no interactions between any of the control factors. Based on these, an orthogonal array (OA) is needed to be selected so that the experimentation can be conducted through. The best fitting OA seems to be $\mathrm{L}_{27}$, which can accommodate 7 factors with 3 levels. This OA suggests a 27 experiments for breaking down the impacts of each control factor on the process while a Full Factorial experimental design will require $3^{7}(2,187)$ experiments for this purpose. Taken these values into consideration, $\mathrm{L}_{27}$ based experimental design with corresponding results have put together in Table A.1 in Appendix, where main statistics, mean, standard deviation and signal-to-noise ratio, are also included.

Table 2. Response Table for Signal to Noise Ratios

\begin{tabular}{cccccccc}
\hline Level & A & B & C & D & E & F & G \\
\hline $\mathbf{1}$ & $-106,6$ & $-106,6$ & $-106,4$ & $-106,2$ & $-106,2$ & $-105,7$ & $-106,7$ \\
$\mathbf{2}$ & $-106,6$ & $-106,4$ & $-106,4$ & $-106,2$ & $-106,5$ & $-106,7$ & $-106,5$ \\
$\mathbf{3}$ & $-106,6$ & $-106,7$ & $-107,0$ & $-107,4$ & $-107,0$ & $-107,3$ & $-106,6$ \\
Delta & 0,0 & 0,3 & 0,6 & 1,2 & 0,8 & 1,6 & 0,2 \\
Rank & 7 & 5 & 4 & 2 & 3 & 1 & 6 \\
\hline
\end{tabular}

The impact of each control factor is determined with ANOVA and impacts are ranked from high-to-low, then a decision is made on either eliminating/revising some factors etc. The ANOVA results are summarised on Table 2 and 3 based on main effect and signal to noise ratios, respectively.

Table 3. Response Table for Means

\begin{tabular}{cccccccc}
\hline Level & A & B & C & D & E & F & G \\
\hline $\mathbf{1}$ & 216219 & 215829 & 210876 & 205182 & 206222 & 193073 & 218064 \\
$\mathbf{2}$ & 215020 & 210243 & 209307 & 204051 & 213281 & 217826 & 212127 \\
$\mathbf{3}$ & 214629 & 219797 & 225685 & 236635 & 226365 & 234969 & 215677 \\
Delta & 1590 & 9554 & 16377 & 32584 & 20143 & 41896 & 5937 \\
Rank & 7 & 5 & 4 & 2 & 3 & 1 & 6 \\
\hline
\end{tabular}

These experiments suggest that the factors that are statistically significant affecting the total relevant cost are holding cost (F), minimum order quantity (D), set up cost (E) at $95 \%$ confidence level calculated with ANOVA for RLUC heuristic. The optimum parameter 
setting can be concluded as $\mathrm{A}_{3} \mathrm{~B}_{2} \mathrm{C}_{2} \mathrm{D}_{2} \mathrm{E}_{1} \mathrm{~F}_{1} \mathrm{G}_{2}$ based on Table 2 and 3 . The optimum setting is validated with a confirmation experiment repeated 5 times and averaged in $\$ 174678$. This clearly confirms that the configuration of control factors are the best based on provided information.

These results with TDOE suggest the minimum cost value predicted as $\$ 157,916$. There have been 7 parameters having affected on total relevant cost in this model. This approach was applied to an instance of 150 periods at stochastic demand and price problems using rolling horizons of various lengths. Then, CB, RLUC and RSM are compared one another with respect to the statistics provided in Table 4, where Mean Square Error (MSE), Normalised Mean Square Error (NMSE), Mean Absolute Error (MAE), maximum and minimum absolute (Min Abs, and Max Abs) error and $r$ (correlation co-efficiency) indexes are presented in the rows and the three respected methods are shown on the columns. It is evident that the middle column indicates the lowest error levels of corresponding rows and the highest value for $r$ index, where the error is to be minimised and $r$ is to be maximised up to 1 . This concludes that RLUC outperforms the other two methods; hence, we will rely on RLUC in the remaining investigation.

Table 4. The comparative statistics for $C B, R L U C$ and $R S M$ heuristics

\begin{tabular}{cccc}
\hline Performance & $\boldsymbol{C B}$ & $\boldsymbol{R} \boldsymbol{B} \boldsymbol{C}$ & $\boldsymbol{R S M}$ \\
\hline MSE & 163911229.40 & 64380981.59 & 77503945.29 \\
NMSE & 0.1824 & 0.0518 & 0.0680 \\
MAE & 10096.46 & 6290.36 & 7362.40 \\
Min Abs Error & 674.14 & 570.25 & 645.34 \\
Max Abs Error & 33387.14 & 16782.73 & 15933.61 \\
$r$ & 0.9144 & 0.9782 & 0.9756 \\
\hline
\end{tabular}

\subsection{Back Propagation (BP)}

The second method used for estimation of total system cost constituted of SDLS problems. As mentioned before, ANN models are used to develop simpler, stronger and satisfactory estimation method since the methodologies used are so complicated that one faces so much hardship in this process. A FF-NN model is identified and trained with back propagation algorithm using 102 training data points and tested with another 27 test samples. The code development is made in Java programming language using the configuration and parametric setup provided in Table 5 .

The best result was obtained by the input parameters above. The FF-NN model requires 7 input nodes and 1 output node as part of problem description. The number of hidden nodes has been worked out with trial-and-error since there is no commonly used method for determining the number of hidden nodes to use in a back propagation neural network (FFNN) model [37]. The primarily experimentation was used for this purpose, and realised that the lowest mean square error (MSE) values gained with the combinations of 7-3-1, 7-4-1, 7-81 , where the triples represent input-hidden-output nodes, respectively. It is decided that the most suitable architecture is 7 neurons in input layer, 4 neurons in hidden layer, 1 neuron in output layer. 
Table 5. $F F-N N(\mathrm{BP})$ configuration settings

\begin{tabular}{ll}
\hline Parameter & value \\
\hline Learning rate & 0.01 \\
Number of neurons in output layer & 1 \\
Momentum & 0.9 \\
Activation function & Sigmoid \\
Error margin & 0.01 \\
Epochs & $10000-50000$ \\
Number of Hidden layers & 1 \\
Number of neurons in input layer & 7 \\
Number of neurons in hidden layer & 4 \\
\hline
\end{tabular}

The minimum cost with $B P$ is calculated as $\$ 185,485$ for $A_{3} B_{2} C_{2} D_{1} E_{1} F_{1} G_{2}$ factor combination, where holding cost is 0.5 and minimum order quantity is 100 . The difference between BP predicted and real values is $\$ 10,807$ while the difference between TDOE and real values is $\$ 16,762$.The experimental data set (which is used in TDOE input) is also used in this second model of prediction.

\subsection{FF-NN model trained with Genetic Algorithm (GA)}

GA has been used for further training of FF-NN model to improve the accuracy of its prediction. As described in Section 2.3 and sketched in Figure 2, GA has been taken aboard for better training and more accurate prediction. The implementation of GA for learning purpose consists of the fundamental characteristics explained earlier. The first step is to generate a population of solutions in which each solution is described in a chromosome, where a complete set of weights is considered as a chromosome. Research shows that it is not useful to increase population size, because it does not make solving the problem faster. In this study, the population size is set to100. The number of genes on each chromosome corresponds to the number of connection within the complete FF-NN model is $32(7 x 4+4 x 1)$, where 7 input nodes are connected all 4 hidden nodes and all hidden nodes are connected to the output node. Any two chromosomes within the population selected with binary tournament selection operator are crossovered with two-point-crossover operator subject to crossover rate, which is set to 1 in this application, and one of the generated and qualified chromosomes is mutated subject to mutation rate, which is 0.005 . The whole evolution process is run through 10,000 generations. The results are summarised in Table 7 to be compared with those achieved with other models.

\section{4. $\quad$ FF-NN model using Bees Algorithm (BA)}

The last heuristic approach implemented for further training FF-NN model is BA, which has been described before as an optimisation algorithm inspired of the natural foraging behaviour of honey bees to find the optimal solution. Detailed information can be found in Pham et al. [31] and Düğenci [32]. Parameter setting, decided empirically, is shown in Table 6. The number of iteration is 10,000 as the stopping criterion, which is the same as GA. Performance results obtained for RLUC, CB, and RSM by using four modelling methods namely FF-NN, GA, BA, TDOE is given in Table 7. 
Table 6. The parameters of the $B A$

\begin{tabular}{lll}
\hline Bees Algorithm parameters & Symbol & Value \\
\hline Number of scout bees & $N$ & 100 \\
Number of selected sites & $M$ & 20 \\
Number of elite bees & $E$ & 10 \\
Initial patch size & $\gamma$ & 0.1 \\
Number of bees around elite points & $n_{E}$ & 50 \\
Number of bees around other selected points & $n_{M-E}$ & 20 \\
\hline
\end{tabular}

Table 7. Performance results obtained for $R L U C, C B, R S M$

\begin{tabular}{|c|c|c|c|c|c|}
\hline & & $B P$ & $\boldsymbol{G A}$ & $\boldsymbol{B A}$ & TDOE \\
\hline \multirow{6}{*}{$R L U C$} & $\mathrm{ME}$ & -2037.41 & -905.63 & 49.56 & -3084.22 \\
\hline & MAE & 6290.36 & 8218.49 & 5114.25 & 23823.38 \\
\hline & RMSE & 8023.78 & 10029.43 & 6659.90 & 30033.31 \\
\hline & MPE & -0.91 & -0.77 & -0.04 & -2.51712 \\
\hline & MAPE & 2.89 & 3.90 & 2.29 & 11.0093 \\
\hline & $\mathrm{R}^{2}$ & 0.999948 & 0.999912 & 0.999967 & 0.999221 \\
\hline \multirow{6}{*}{$C B$} & $\mathrm{ME}$ & -1596.80 & -417.49 & 1912.51 & -12071.4 \\
\hline & MAE & 10096.45 & 10991.74 & 8111.99 & 20127.25 \\
\hline & RMSE & 12802.78 & 13609.18 & 12575.14 & 25961.51 \\
\hline & MPE & -0.91 & -0.60 & 0.66 & -6.12742 \\
\hline & MAPE & 4.67 & 5.32 & 3.75 & 9.487606 \\
\hline & $\mathrm{R}^{2}$ & 0.999864 & 0.999843 & 0.999868 & 0.999571 \\
\hline \multirow{6}{*}{$R S M$} & $\mathrm{ME}$ & -3105.32 & -976.06 & -1396.43 & -5584.41 \\
\hline & MAE & 7362.40 & 4737.54 & 4052.43 & 22130.04 \\
\hline & RMSE & 8803.63 & 5823.57 & 5621.29 & 31499.74 \\
\hline & MPE & -1.35 & -0.46 & -0.71 & -3.53179 \\
\hline & MAPE & 3.38 & 2.22 & 1.93 & 10.19988 \\
\hline & $\mathrm{R}^{2}$ & 0.999943 & 0.999971 & 0.999974 & 0.999172 \\
\hline
\end{tabular}

Table 7 summarises the experimental results of all 4 models, (i.e. BP, GA, BA and TDOE), using all 3 calculation methods, (i.e. CB, RLUC and RSM). The statistics used are mainly the error measures and the coefficient of determination $\left(\mathrm{R}^{2}\right)$. The error measures are Mean Error (ME), Mean Absolute Error (MAE), Mean Percentage Error (MPE), Root Mean Square Error (RMSE) and Mean Absolute Percentage Error (MAPE). As mentioned above, the error measures are to be minimised and the coefficient of determination is to be maximised up to 1 . As is seen, the best prediction is scored by BA for all 3 calculation methods with respect to all statistical measures, where all error levels remain the lowest and the $\mathrm{R}^{2}$ achieved the highest with BA. The success of the models with CB and RLUC can be ordered as BA, BP, GA and TDOE while GA and BP swap the order with RSM.

Figure 3 presents test results of all FF-NN models in comparison to real simulation results for RLUC calculation method. The solid line indicates the simulated results, while the filled 
squares ( $\square)$ show the performance of BP model, unfilled squares $(\square)$ point out performance of GA model and plus signs (+) represent BA's performance per corresponding test sample. The number of points that overlap on the solid line indicates the test performance of each learning algorithm, where BA scores 24, GA does 12 and BP hits 17 out of 27 . That means BA is $89 \%$, $\mathrm{GA}$ is $44 \%$ and BP is $63 \%$ successful in test results with $99 \%$ confidence level. This concludes that BA is the most successful learning approach over other two in finding the most satisfactory results of the total relevant cost with RLUC method.

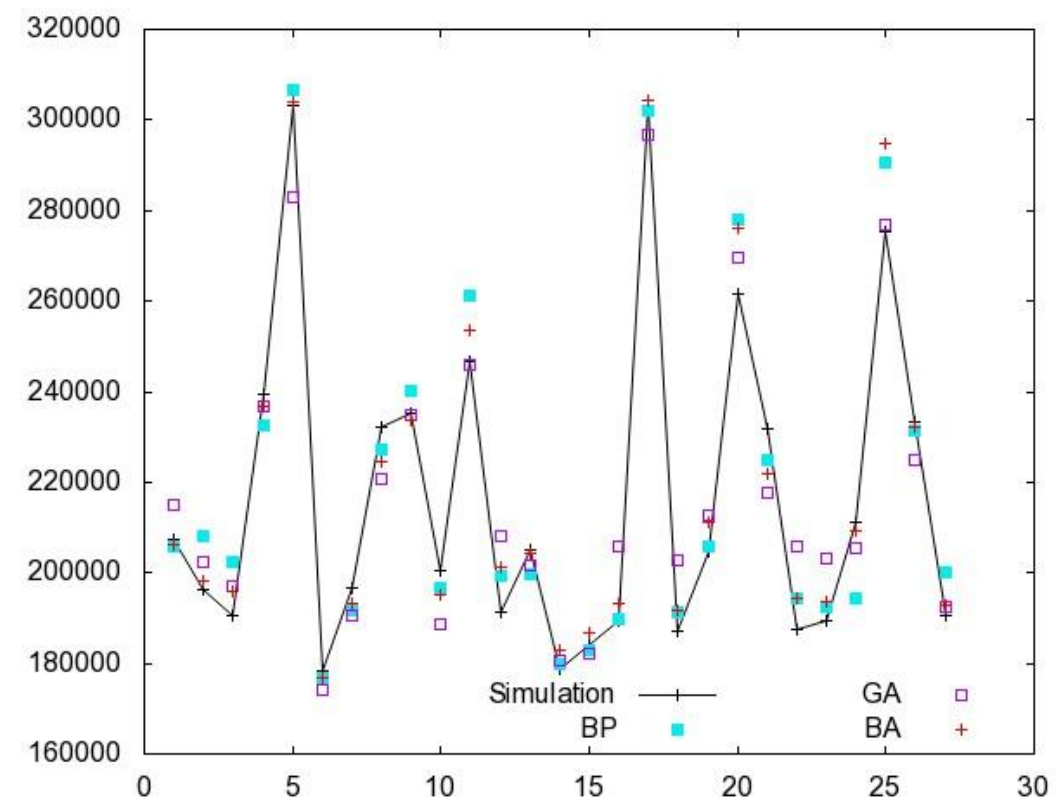

Figure 3 The performance of FF-NN models with BP, GA and BA

\section{Conclusion}

This paper reports an investigation for solving the problem of multiple period single-item SDLS with uncertain and variable demand and price, where holding cost assumed independent of purchasing cost. Since SDLS problem is analytically intractable, heuristics embedded in FF-NN approaches are used to model and solve the problem. We have made an application dealing with multiple period single-item lot sizing problem with lost sale and rolling horizon using FF-NN models trained with BP, GA and BA. The significant factors and their levels identifying the problem and its degree of freedom have been considered as system inputs for RLUC, RSM, and CB cost calculation methods. After a TDOE-based ANOVA, RLUC is found the best of three operating methods with simulation. BA has been found the best of 3 learning heuristic algorithms used to train FF-NN models for lot-sizing problems first time in the literature.

\section{References}

[1] E. Şenyiğit, A new lot sizing heuristic for MRP systems and its analysis, MSc Thesis (2001) Çukurova University.

[2] R. Jans, Z. Degraeve, Meta-heuristics for dynamic lot sizing: a review and comparison of solution approaches. European Journal of Operational Research 177 (2007) 1855-1875. 
[3] H.G. Gören, S. Tunali, R. Jans, A review of applications of genetic algorithms in lot sizing, Journal of Intelligent Manufacturing, Vol. 21, (2010), 575-590.

[4] H.C. Bahl, L.P. Ritzman, J.N.D Gupta, Determining lot sizes and resource requirements: a review, Operations Research 35 (1987) 329-345.

[5] L.K. Gaafar, M.H. Choueiki, A neural network model for solving the lot sizing problem, The International Journal of Management Science, Omega Vol. 28 (2000) 175-184.

[6] U. Atici, Lot-sizing by using artificial neural network. MSc Thesis (2011) Erciyes University.

[7] A. Manikas, Y. Chang, M. Ferguson, BlueLinx can benefit from innovative inventory management methods for commodity forward buys, Omega 37 (2009) 545-554.

[8] E. Şenyiğit, R. Erol, New lot sizing heuristics for demand and price uncertainties with service-level constraint, International Journal of Production Research 48 (2010) 21-44.

[9] N. Megala, N. Jawahar, Genetic algorithm and Hopfield neural network for a dynamic lot sizing problem, International Journal of Advanced Manufacturing Technology 27 (2006) 1178-1191.

[10] G. Marco, T. Marco, Neural networks in production planning and control, Production Planning \& Control 10 (4) (1999) 324 -339.

[11] H.M. Wagner, T.M. Whitin, Dynamic version of the economic lot size model, Management Science (1958) 89-96.

[12] E.A. Silver, H.C. Meal, A heuristic for selecting lot size quantities for the case of a deterministic time varying demand rate and discrete opportunities for replenishment, Production Inventory Management 14 (2) (1973) 64-74.

[13] E.A. Silver, R. Peterson, Decision systems for inventory management and Production Planning (1985) New York: Wiley.

[14] M. Chen, S. C. Sarin, A. Peake, Integrated lot sizing and dispatching in wafer Fabrication, Production Planning \& Control 21 ( 5), (2010) 485-495.

[15] J.J. De Matteis, A.G. Mendoza, An economic lot sizing technique, IBM systems journal 7 (1968) $30-46$.

[16] J. Orlicky, Material Requirement Planning (1974) New York: McGraw Hill.

[17] N.H.M. Radzi, H. Haron, T.I.T. Jahori, Lot Sizing Using Neural Network Approach, Proceedings of the $2^{\text {nd }}$ IMT-GT Regional Conference on Mathematics, Statistic and Applications Universiti Sains Malaysia Penang (2000) June 13-15.

[18] K.J. Anil, J. Mao, K.M. Mohiuddin, Artificial Neural Network: A Tutorial, IBM Almaden Research Centre, Michigan, Michigan State University (1996) 1-14.

[19] E.H.L. Aarts, M.F. Reijnhoudt, H.P. Stehouwer, J. Wessels, A Novel Decomposition Approach for online lot-sizing. European Journal of Operational Research 122 (2000) 339-353.

[20] E. Öztemel, M.E. Aydin, An artificial neural network-based experimental design method", Journal of Turkish Environment and Engineering, (1996) 20(2).

[21] J.H. Bookbinder, J.Y. Tan, Strategies for the probabilistic lot sizing problem with service level constraints, Management Science 34 (1988) 1096-1108.

[22] S.A.Tarim, B.G. Kingsman, The stochastic dynamic production/inventory lot sizing problem with service-level constraints, International Journal of Production Economics 88 (2004) 105-119.

[23] S.A. Tarim, B.G. Kingsman, Modelling and computing ( $\mathrm{Rn}, \mathrm{Sn})$ policies for inventory systems with nonstationary stochastic demand, European Journal of Operational Research 174 (2006) 581-599.

[24] S.A. Tarim, B.M. Smith, Constraint programming for computing non-stationary (R, S) inventory systems, European Journal of Operational Research 189 (2008) 1004-1021. 
[25] C. Li, F. Liu, H. Cao, Q. Wang, A stochastic dynamic programming based model for uncertain production planning of re-manufacturing system, International Journal of Production Research 47 (13) (2009) 3657 3668 .

[26] P. Brandimarte, Multi-item capacitated lot-sizing with demand uncertainty, International Journal of Production Research 44 (15) (2006) 2997 - 3022.

[27] K.Y. Chan, C.K. Kwong, X.G. Luo, Improved orthogonal array based simulated annealing with interaction analysis between variables for design optimization, Expert Systems with Applications 36(4) (2009) 7379-7389.

[28] K.Y. Chan, C.K. Kwong, H. Jiang, M.E. Aydin, T.C. Fogarty, A new orthogonal array based crossover, with analysis of gene interactions, for evolutionary algorithms and its application to car door design, Expert Systems with Applications 37(5) (2010) 3853-3862.

[29] X. Yao, A review of evolutionary artificial neural networks, International Journal of Intelligent Systems, 8 (1993) 539-567.

[30] Y. Da, G. Xiurun, An improved PSO-based ANN with simulated annealing technique, Neurocomputing, 63 (2005) 527-533.

[31] D. T. Pham, S. Otri, A. Ghanbarzadeh, E. Koc, Application of the bees algorithm to the training of learning vector quantisation networks for control chart pattern recognition. Proceedings of the Information and Communication Technologies (ICTTA'06) 1624-1629 (2006). Syria.

[32] M. Düğenci, Using the bee's algorithm for artificial neural Networks training and the control application of wastewater treatment plant (2007) Thesis (PhD). Sakarya University.

[33] D.Karaboga, B. Akay, A Survey: Algorithms Simulating Bee Swarm Intelligence, Artificial Intelligence Review 31 (1) (2009) 68-85.

[34] M. Farooq, Bee-inspired protocol engineering: From nature to networks. Berlin, Heidelberg, (2008) Germany: Springer.

[35] D. T. Pham, A. Afify, E.Koc, Manufacturing cell formation using the Bees Algorithm. IPROMS'2007 Innovative Production Machines and Systems Virtual Conference Cardiff (2007) UK.

[36] D.T. Pham, A. Ghanbarzadeh, E. Koç, S. Otri, S. Rahim, M. Zaid, The Bees algorithm-A Novel Tool for Complex Optimisation Problems, IPROMS'2006 Intelligent Production Machines and Systems, (2006) 454-459.

[37] C.C. Chiu, C.T. Su, G.S. Yang, J-S. Huang, S-C. Chen, N-T. Cheng, Selection of optimal parameters in gas-assisted injection moulding using a neural network model and the Taguchi method, International Journal of Quality Science 2 (2) (1997) 106-120. 


\section{Appendix}

Table A.1. OA design table including details of $L_{27}$ and corresponding repetitions of the experimental results.

\begin{tabular}{|c|c|c|c|c|c|c|c|c|c|c|c|c|c|}
\hline \multirow[b]{2}{*}{ Trial No } & \multicolumn{7}{|c|}{ Control factor } & \multicolumn{3}{|c|}{ Cost } & \multirow[b]{2}{*}{ Avg. (Cost) } & \multirow[b]{2}{*}{ Std.Dev. } & \multirow[b]{2}{*}{$\mathrm{S} / \mathrm{N}$} \\
\hline & $\mathbf{A}$ & B & $\mathbf{C}$ & D & $\mathbf{E}$ & $\mathbf{F}$ & $\mathbf{G}$ & Y1 & Y2 & Y3 & & & \\
\hline 1 & 1 & 1 & 1 & 1 & 1 & 1 & 1 & 160 & 180070 & 179990 & 178406,67 & 2811,98 & 05,029 \\
\hline 2 & 1 & 1 & 1 & 1 & 2 & 2 & 2 & 30 & 30 & 90 & 00 & 18 & \\
\hline 3 & 1 & 1 & 1 & 1 & 3 & 3 & 3 & & 233830 & 233360 & 2320 & & \\
\hline 4 & 1 & 2 & 2 & 2 & 1 & 1 & 1 & 90 & 188610 & 188540 & 184246,67 & & \\
\hline 5 & 1 & 2 & 2 & 2 & 2 & 2 & 2 & & 209 & 0 & 2049 & & \\
\hline 6 & 1 & 2 & 2 & 2 & 3 & 3 & 3 & & 197790 & 196890 & 1 & & \\
\hline 7 & 1 & 3 & 3 & 3 & 1 & 1 & 1 & 18 & 208190 & 203340 & 200 & 94 & -106 \\
\hline 8 & 1 & 3 & 3 & 3 & 2 & 2 & 2 & 227 & 249120 & 241510 & 00 & 27 & -10 \\
\hline 9 & 1 & 3 & 3 & . & 3 & 3 & 3 & 303540 & 330990 & 317840 & 67 & 01 & -110 \\
\hline 10 & 2 & 1 & 2 & 3 & 1 & 2 & 3 & 224 & 234940 & 230990 & 230050,00 & 54 & -107 \\
\hline 11 & 2 & 1 & 2 & 3 & 2 & 3 & 1 & & 259 & $26^{\circ}$ & & & \\
\hline 12 & 2 & 1 & 2 & 3 & 3 & 1 & 2 & & 204750 & 204 & & & -10 \\
\hline 13 & 2 & 2 & 3 & 1 & 1 & 2 & 3 & & 209100 & 207320 & 67 & & -10 \\
\hline 14 & 2 & 2 & 3 & 1 & 2 & 3 & 1 & & 224160 & 227690 & 2223 & & -106 \\
\hline 15 & 2 & 2 & 3 & 1 & 3 & 1 & 2 & 192440 & 210870 & 210120 & 204476,67 & 10430,80 & $-106,22$ \\
\hline 16 & 2 & 3 & 1 & 2 & 1 & 2 & 3 & & 197790 & 196890 & & & -10 \\
\hline 17 & 2 & 3 & 1 & 2 & 2 & 3 & 1 & & 226320 & 230150 & & & -106 \\
\hline 18 & 2 & 3 & 1 & 2 & 3 & 1 & 2 & 182290 & 201520 & 205540 & 196450,00 & 6,56 & -105 \\
\hline 19 & 3 & 1 & 3 & 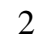 & 1 & 3 & 2 & & 221250 & 224290 & & & -106 \\
\hline 20 & 3 & 1 & 3 & 2 & 2 & 1 & 3 & 50 & 201 & 200 & 19 &, 44 & -105 \\
\hline 21 & 3 & 1 & 3 & 2 & 3 & 2 & 1 & & 232600 & 231670 & 226803,33 & & $-107,118$ \\
\hline 22 & 3 & 2 & 1 & 3 & 1 & 3 & 2 & 238 & 247860 & 254060 & 246813,33 & ,69 & $-107,85$ \\
\hline 23 & 3 & 2 & 1 & 3 & 2 & 1 & 3 & 176820 & 191150 & 193740 & 187236,67 & 9113,57 & $-105,455$ \\
\hline 24 & 3 & 2 & 1 & 3 & 3 & 2 & 1 & 235000 & 251810 & 249670 & 245493,33 & 9150,27 & $-107,805$ \\
\hline 25 & 3 & 3 & 2 & 1 & 1 & 3 & 2 & 19 & 198720 & 200070 & 197996,67 & 2514,29 & $-105,934$ \\
\hline 26 & 3 & 3 & 2 & 1 & 2 & 1 & 3 & & 195580 & 196380 & 190393,33 & 9684,65 & $-105,601$ \\
\hline$\rightarrow$ & 3 & 3 & 2 & 1 & J & 2 & 1 & 210700 & 226440 & 225120 & 220753,33 & 8731,42 & $-106,883$ \\
\hline
\end{tabular}

